\title{
Síndrome de Tako-Tsubo (discinesia apical transitoria). Un síndrome que simula un infarto de miocardio
}

\author{
I. SALAVERRÍA GARZÓN, M. VILLASEÑOR NAVAS, S. SÁNCHEZ HERRERA, \\ L. MARTÍNEZ ELBAL ${ }^{1}$
}

Servicio de Medicina Intensiva y ${ }^{1}$ Hemodinámica. Clínica Ruber. Madrid

\section{RESUMEN}

El syndrome de Tako-Tsubo o disquinesia apical transitoria, es un síndrome que puede simular un evento coronario agudo. Se caracteriza por dolor precordial anginoso con cambios en el ST y onda T en precordiales, no obstrucción coronaria en la coronariografía y una característica disquinesia antero-apical del ventrículo izquierdo que se normaliza en unos pocos días. Este síndrome ha sido descrito en Japón, pero pocos casos has sido reportados in Occidente. Nosotros presentamos un caso de síndrome de Tako-Tsubo después de una cirugía de tiroides.

PALABRAS CLAVE: Disquinesia apical transitoria. Síndrome Tako-Tsubo. Infarto de miocardio. Cirugía de tiroides.

TAKO-TSUBO SYNDROME (TRANSIENT APICAL DYSKINESIA). AN ENTITY THAT CAN MIMIC A MYOCARDIAL INFARCTION

\begin{abstract}
Tako-Tsubo syndrome or transient apical dyskinesia, is an entity that can mimic an acute coronary event. It is characterized by anginal chest pain with ST-T changes in precordial leads, no coronary obstruction on angiography and a characteristic left ventricular antero-apical dyskinesia that recovers to normal in a few days. This syndrome has been described in Japan, but few cases have been reported in Western countries. We report a case of Tako-Tsubo syndrome after thyroid surgery.
\end{abstract}

KEY WORDS: Transient left ventricular apical ballooning. Tako-Tsubo syndrome. Myocardial infarction. Thyroid surgery.

Salaverría Garzón I, Mariano Villaseñor M, Sánchez Herrera S, Martínez Elbal L. Síndrome de Tako-Tsubo (discinesia apical transitoria). Un síndrome que simula un infarto agudo de miocardio. An Med Interna (Madrid) 2008; 25: 20-22.

\section{INTRODUCCIÓN}

El síndrome de discinesia apical transitoria, o síndrome de Tako-Tsubo, es una entidad clínica descrita por primera vez por Dote, Sato y cols. en 1991 (1) en Japón y de la que tan sólo existen pequeñas series descritas.

El síndrome de Tako-Tsubo suele comenzar después de un cuadro de estrés importante, físico o psíquico, y tanto la clínica como el electrocardiograma y el ecocardiograma inicial, simulan un infarto agudo de miocardio apical (IAM). Sin embargo, en el síndrome de Tako-Tsubo no existen alteraciones coronarias significativas y la contractilidad ventricular se normaliza totalmente en unos pocos días.

Es posible que este síndrome sea más frecuente de lo que creemos, pero que pase desapercibido. Por ello, es importante conocer la existencia de este cuadro, para poder sospecharlo y diagnosticarlo, ya que el tratamiento y pronóstico son distintos al del IAM por trombosis coronaria.

\section{CASO APORTADO}

Presentamos el caso de una mujer de 57 años, con antecedentes de hipertensión arterial, hiperlipemia, fumadora y sin antecedentes coronarios conocidos. A la paciente se le realizó una hemitiroidectomía con extirpación de adenoma paratiroideo e ingresó en UCI, despierta y extubada, para control del postoperatorio inmediato. A las pocas horas del ingreso, la paciente presentó un episodio de dolor interescapular, sin disnea ni otra sintomatología, no mostrando cambios electrocardiográficos (Fig. 1) y autolimitándose el dolor en pocos minutos. En el ECG de control que se realizó a la mañana siguiente (Fig. 2), apareció un descenso del punto J V4-V6 y onda T negativa en cara antero-lateral extensa e inferior. En el ecocardiograma urgente se apreció una hipoquinesia severa del ápex, con el resto de segmentos normales y una FEVI 50\%. En la coronariografía no se objetivaron lesiones coronarias y la ventriculografía mostró aquinesia apical. En la seriación enzimática, el pico de Troponina I fue 0,48 ng/ml y el de CPK 206 UI/l. La paciente evolucionó satisfactoriamente, no presentando complicaciones añadidas desde el punto de vista cardiológico y siendo dado de alta a domicilio a los pocos días

Trabajo aceptado: 27 de agosto de 2007 
de la intervención. Se repitió el ecocardiograma de forma ambulatoria a los 14 días, encontrando un ventrículo izquierdo de contractilidad normal y FEVI del $74 \%$.

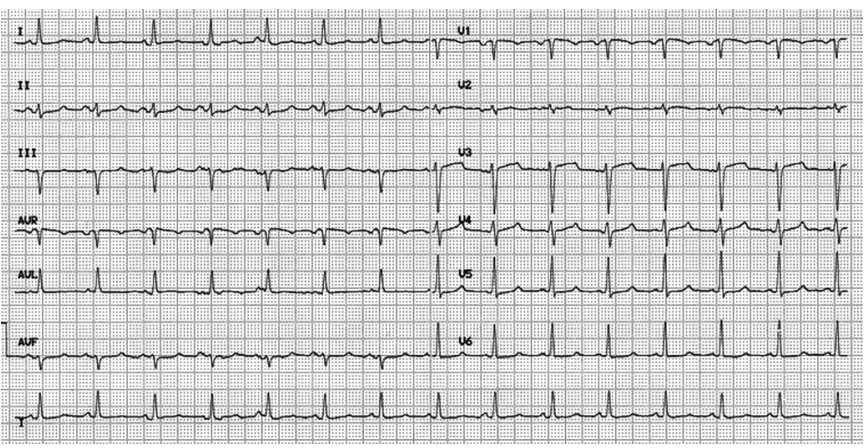

Fig. 1. ECG de ingreso en UCI (postcirugía).

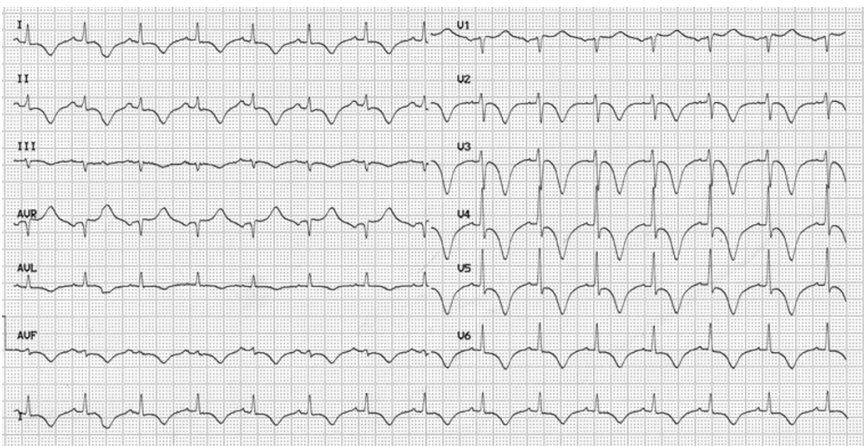

Fig. 2. ECG a las 12 horas de ingreso en UCI.

\section{DISCUSIÓN}

El síndrome de discinesia apical transitoria o síndrome de Tako-Tsubo se caracteriza por (2): aquinesia o discinesia transitoria que afecta principalmente al ápex del ventrículo izquierdo, ausencia de lesiones coronarias y cambios electrocardiográficos con elevación del segmento ST o inversión de la onda T.

La incidencia no está clara y no se sabe cuantos de los IAM que tienen coronarias normales $(6 \%)$, podrían ser síndromes de Tako-Tsubo.

Este síndrome afecta principalmente a mujeres (4:1) (3), de edad media-avanzada (media 62-75 años).

Caso siempre existe un desencadenante que suele ser un episodio de estrés físico, un ejercicio físico inhabitual, un estrés emocional agudo (4), o una cirugía (5) como en el caso que presentamos.

La fisiopatología todavía es desconocida. Como hipótesis se han propuesto: una elevación brusca e intensa de catecolaminas (que produciría vasoconstricción de la microvasculatura cardíaca), espasmos coronarios (aunque sólo el 21\% de los pacientes presentan vasoespasmos con acetilcolina), obstrucción dinámica medioventricular y la existencia de una coronaria descendente anterior con gran segmento recurrente (6).

La presentación clínica del cuadro es similar a la de un síndrome coronario agudo. Puede iniciarse con dolor torácico
(53-71\%) de característica e irradiación similares a la cardiopatía isquémica, con disnea (7-20\%) y más raramente con shock cardiogénico $(5 \%)$.

El ECG inicial puede ser similar al de un síndrome coronario agudo con elevación del ST. Presentan elevación del ST en precordiales (90\%), onda $\mathrm{T}$ negativa en precordiales (44\%), onda Q (15-27\%), siendo rara la imagen especular en cara inferior. Los cambios electrocardiográficos pueden durar días o semanas y evolucionan hacia desaparición de la onda Q (90-100\%), normalización del ST y presencia de ondas T negativas y profundas (84-97\%). Estos cambios electrocardiográficos se explican por la gran diferencia entre la repolarización de la zona apical discinética y la basal hiperquinética.

En la ecografia realizada en el momento inicial, aparece una disquinesia o aquinesia del apex del ventrículo izquierdo, con normo o hiperquinesia basal.

Las enzimas cardíacas pueden elevarse levemente (CPK en el 56\%, troponina casi en el 100\%), pero esta elevación es de escasa cuantía y con una curva que evoluciona rápidamente hacia la normalización, sin seguir la curva lenta típica del IAM.

La coronariografía no muestra lesiones significativas coronarias. La ventriculografía (con la hipoquinesia apical ya descrita) da una imagen típica, que recuerda a una vasija de cuello angosto y base globular y ancha como las que en Japón se utiliza para capturar pulpos, denominadas Tako-Tsubo y que han dado nombre a este síndrome.

Como complicaciones pueden aparecer arritmias ventriculares $(9 \%)$, bradicardia severa $(10 \%)$, algún grado insuficiencia cardíaca (22\%), estenosis subaórtica dinámica, shock cardiogénico $(15 \%)$ e incluso se ha descrito un caso de accidente cerebrovascular de origen embólico.

La recuperación clínica y la mejoría hemodinámica, normalmente comienza al 2-3 ${ }^{\circ}$ día, los cambios en el ECG pueden durar días o semanas, y los cambios ecográficos se normalizan en días o semanas (3-31 días).

La evolución del cuadro normalmente es benigna, con una mortalidad del $1 \%$ y la recurrencia es rara, aunque varía del 0 $8 \%$ según las series.

La hipovolemia, los inotropos y el balón de contrapulsación intraaórtico empeoran la obstrucción intraventricular dinámica y con ello disminuyen el gasto cardíaco, por lo que deben evitarse. Pueden utilizarse, con cuidado, los beta-bloqueantes para disminuir este gradiente ventricular. El manejo de la volemia debe de ser cuidadoso para evitar aumentar el gradiente ventricular por hipovolemia y tratar de no empeorar la posible insuficiencia cardíaca por hipervolemia.

Distinguir, en urgencias, con la clínica y el ECG, un síndrome de Tako-Tsubo de un síndrome coronario agudo por trombosis coronaria puede ser difícil. Existe una pequeña serie de 13 casos (8), que sugiere que hay 2 signos que orientan al un síndrome de Tako-Tsubo. Uno, que la elevación del ST en V4-V6 es mayor que en V1-V3, con ausencia de Q anormal. Y dos, que no existen los cambios especulares en la cara inferior (descenso del ST en esas derivadas). Aunque estos signos electrocardiográficos todavía no han sido suficientemente contrastados como para poder distinguir entre ambos síndromes, en el caso de existir, debería valorarse realizar un ecocardiograma urgente que confirme la aquinesia apical o antero-apical y entonces optar por una coronariografía en lugar de por tratamiento fibrinolítico, para evitar fibrinolisar un síndrome de Tako-Tsubo. 


\section{Bibliografía}

1. Dote K, Sato H, Tateishi H, Uchida T, Ishihara M. Myocardial stunning due to simultaneous multivessel coronary spasms: A review of 5 cases. J Cardiol 1991; 21: 203-14

2. Nyui N, Yamanaka O, Nakayama R, Sawano M, Kawai S. "Tako-Tsubo" transient ventricular dysfunction: A case report. Jpn Circ J 2000; 64: 715-9.

3. Bybee KA, Kara T, Prasad A, Lerman A, Barsness GW, Wright RS, Rihal CS. Systematic review: Transient left ventricular apical ballooning: a syndrome that mimics ST-segment elevation myocardial infarction. Ann Intern Med 2004; 141: 858-65.

4. Gallego Page JC, Lafuente Gormaz C, Domínguez Rodríguez P, Chafer Rudilla M, Fuentes Manso R, Aguilera Saldana M. Disfunción ventricular transitoria tras estrés emocional. Rev Esp Cardiol 2004; 57: 1124-7.
5. Pueo EM, Diarte de Miguel JA, Chopo JM, Placer LJ. Síndrome de discinesia apical transitoria. A propósito de un caso. Rev Fed Arg Cardiol 2003; 32: 415-8.

6. Ibanez B, Navarro F, Farre J, Marcos-Alberca P, Orejas M, Rabago R, et al. Asociación del síndrome Tako-Tsubo con la arteria coronaria descendente anterior con extensa distribución por el segmento diafragmático. Rev Esp Cardiol. 2004; 57: 209-16.

7. Gaspar J, Gomez Cruz RA. Síndrome Tako-Tsubo (discinesia anteroapical transitoria): primer caso descrito en América Latina y revisión de la literatura. Arch Cardiol Mex. 2004; 74: 205-14.

8. Ogura R, Hiasa Y, Takahashi T, Yamaguchi K, Fujiwara K, Ohara Y, et al. Specific findings of the standard 12-lead ECG in patients with "Tako-Tsubo" cardiomyopathy: Comparison with the findings of acute anterior myocardial infarction. Circ J 2003; 67: 687-90. 